\title{
Improving the performance of the Actinic Inspection Tool with an optimized alignment procedure
}

\author{
Iacopo Mochi*a ${ }^{\text {a }}$ Kenneth A. Goldberg ${ }^{\mathrm{a}}$, Patrick Naulleau ${ }^{\mathrm{a}}$, Sungmin Huh $^{\mathrm{b}}$ \\ ${ }^{a}$ Lawrence Berkeley National Laboratory, One Cyclotron Road, Berkeley, CA, USA 94720; \\ ${ }^{\mathrm{b}}$ SEMATECH, 255 Fuller Road Suite 309, Albany, NY 12203
}

\begin{abstract}
Extreme ultraviolet (EUV) microscopy is an important tool for the investigation of the performance of EUV masks, for detecting the presence and the characteristics of defects, and for evaluating the effectiveness of defect repair techniques. Aerial image measurement bypasses the difficulties inherent to photoresist imaging and enables high data collection speed and flexibility. It provides reliable and quick feedback for the development of masks and lithography system modeling methods.

We operate the SEMATECH Berkeley Actinic Inspection Tool (AIT), a EUV microscope installed at the Advanced Light Source at Lawrence Berkeley National Laboratory. The AIT is equipped with several highmagnification Fresnel zoneplate lenses, with various numerical aperture values, that enable it image the reflective mask surface with various resolution and magnification settings. Although the AIT has undergone significant recent improvements in terms of imaging resolution and illumination uniformity, there is still room for improvement.

In the AIT, an off-axis zoneplate lens collects the light coming from the sample and an image of the sample is projected onto an EUV-sensitive CCD camera. The simplicity of the optical system is particularly helpful considering that the AIT alignment has to be performed every time that a sample or a zoneplate is replaced. The alignment is sensitive to several parameters such as the lens position and orientation, the illumination direction and the sample characteristics. Since the AIT works in high vacuum, there is no direct access to the optics or to the sample during the alignment and the measurements. For all these reasons the alignment procedures and feedback can be complex, and in some cases can reduce the overall data throughput of the system. In this paper we review the main strategies and procedures that have been developed for quick and reliable alignments, and we describe the performance improvements we have achieved, in terms of aberration magnitude reduction.
\end{abstract}

Keywords: EUV, zoneplate, alignment, mask inspection, aerial image, aberrations

\section{INTRODUCTION}

Masks quality assessment is an essential step in the EUV lithography process. It is necessary to look for mask defects and assess their printability, be they phase defects caused by multilayer imperfections, or defects and contamination in the patterned absorber. Following defect discovery and characterization it might be necessary to evaluate the quality of the eventual repair. It is also essential to characterize the mask and the pattern in terms of contrast, pattern roughness, and, ultimately, to evaluate the quality of the features that will be printed on the wafer. All these controls can be carried out by means of a mask inspection tool that collects the aerial image of the mask without the need of actually printing the pattern. Continuous-intensity images collected by an aerial image microscope are not degraded by the limited quality of photoresist and they can be used to predict the imaging performance of the mask.

The use of EUV inspection tools is bound to increase in the near future when the technology for EUV lithography becomes ready for mass production. While the availability of commercial EUV mask tools is still several years away, we operate a research prototype for the advanced development and characterization of EUV masks. The SEMATECH Berkeley Actinic Inspection Tool (AIT) is a synchrotron-based zoneplate microscope installed at the Advanced Light Source (ALS) at Lawrence Berkeley National Laboratory.

In recent years the AIT has been substantially upgraded ${ }^{1}$ and its performance has increased dramatically in terms of data throughput, resolution, and repeatability. To further improve the image quality of the tool we have concentrated on the 
reduction of optical aberrations. The alignment of the optical imaging system minimized aberrations that degrade the image quality. The effects of small aberrations are difficult to assess by eye, but can be clearly evident from analysis of the collected data ${ }^{2}$. Every time a lens is changed or repositioned, a careful alignment must be performed to minimize the aberrations in the portion of the field of view we are interested in.

Several techniques have been proposed and developed to approach the problem of the in situ aberration retrieval for optical systems in the field of micro-lithography ${ }^{3,4,5}$. Most of them rely on the analysis of resist-printed images, and utilize specific reticle patterns. These techniques deduce wavefront information from the intensity profile of the point spread function ${ }^{6}$.

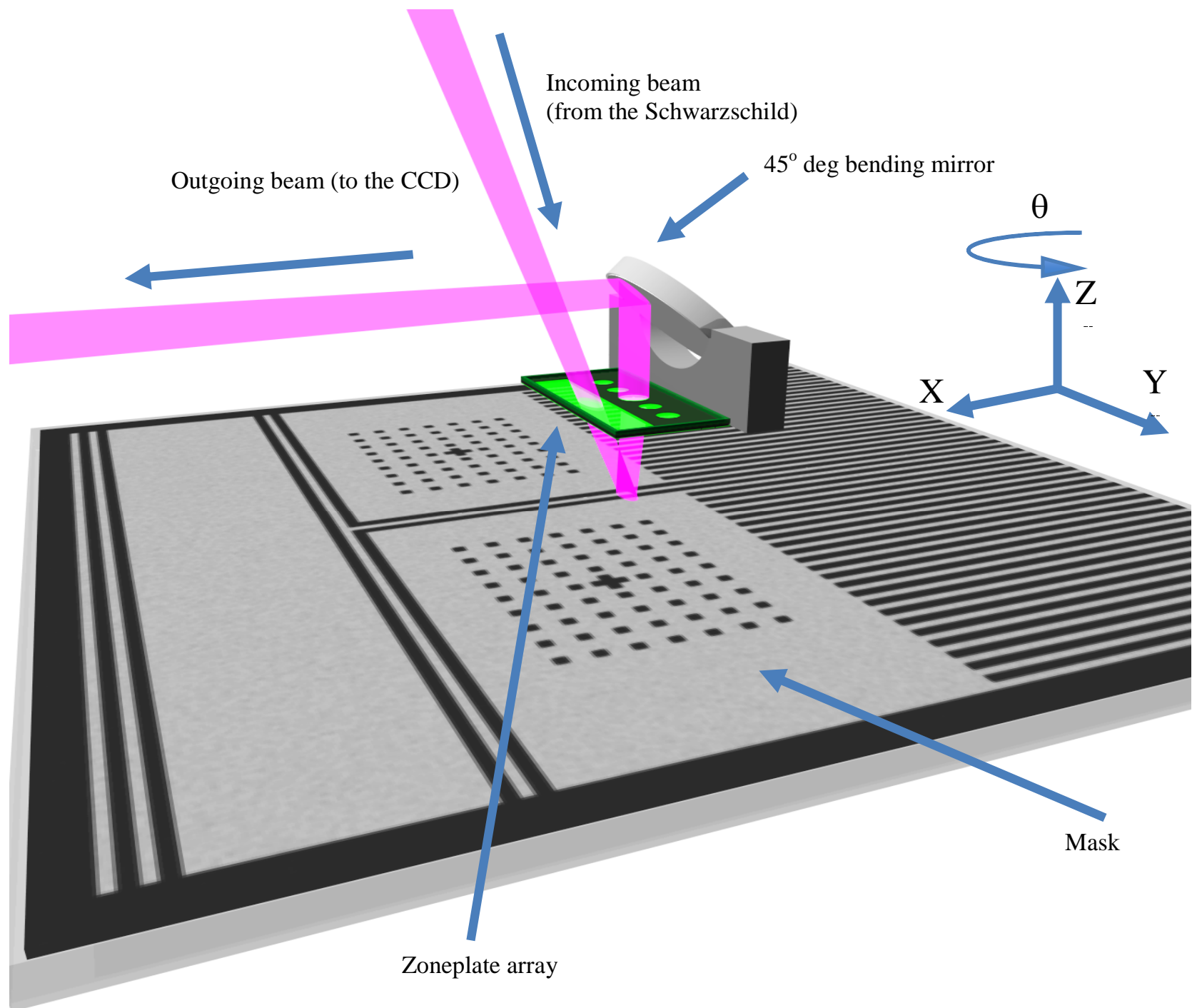

Figure 1 AIT imaging optics schematic drawing. The beam coming from the Schwarzschild objective is projected onto the mask through a transparent window and illuminates the features of interest. An off-axis zoneplate lens that forms an image of the mask on a CCD collects the reflected light.

Similarly, we need a rapid and reliable method to maintain the alignment of the AIT. To detect and minimize the fielddependent and alignment-sensitive aberrations in the AIT imaging, we developed a simple algorithm to measure the distribution of aberrations across the observable field of view. The aberration evaluation is done by studying the change of shape in specific features on the mask, through focus. Our method is based on measurements of the field-dependent aberrations, which vary across the observable field of view, and comparison with the predicted behavior of the system. We rely on the variation in the observable aberrations to guide the adjustment of the available degrees of freedom. 
Ultimately, we position the relatively small aberration-corrected sweet spot (approximately 2-8 $\mu \mathrm{m}$ ) within the center of the field of view. In this way the method goes beyond the analysis of a single field-centered PSF to calculate our position within the alignment phase space. Thus, the measured displacement of the aberration map guides the alignment correction toward the goal.

\subsection{Alignment of the Actinic Inspection Tool}

The AIT is equipped with five zoneplate objective lenses that provide different magnifications (between 680 and $1000 \times$ ) and numerical apertures (NA values between 0.0625 and 0.0875 provide an equivalent resolution to 0.25 to $0.35 \mathrm{NA}, 4 \times$ steppers).

The most frequently used zoneplate lens has an NA of 0.0875 , a focal length $(f)$ of $0.750 \mathrm{~mm}$, and a magnification ratio $(M)$ of approximately 907. In this configuration, the 2048 pixel-wide CCD maps a viewable area of approximately $30-\mu \mathrm{m}$ wide. The measurements and simulations presented here were conducted in this configuration, yet the method may easily be applied to any other configuration.

A bending-magnet beamline focuses narrow-band light onto the mask. A monochromator provides a bandwidth $\lambda / \Delta \lambda$ of approximately 1450. A Kirkpatrick-Baez (KB) mirror pair bring the beam to an intermediate focus, and a multilayercoated Schwarzschild objective provides $20 \times$ demagnification and a $6^{\circ}$ illumination angle to an upward-facing mask. The reflected light is collected by an off-axis zoneplate lens and projected onto an EUV CCD camera after being reflected by a 45 degrees multilayer mirror as shown in Fig. 1. The whole system is maintained in a clean vacuum environment with a base pressure of $10^{-6}$ Torr to allow the propagation of the EUV light and reduce carbon contamination.

We control several degrees of freedom to align the system. Adjustable parameters include: the angle of the incoming beam, the zoneplate position and rotation angle $(x, y, z, \theta)$, and the vertical angle of the $45^{\circ}$ turning mirror that follows the zoneplate and steers the beam horizontally toward the CCD.

We modeled the AIT with Zemax ${ }^{\circledR}$ to characterize the field-dependent wavefront aberrations in an ideal alignment state. The off-axis geometry of the optical system angularly separates the zoneplate's undiffracted zero-order light from the first order light that forms the image, yet it complicates the geometry by introducing a $3.5^{\circ}$ tilt in the focal plane ${ }^{7}$. Appropriately compensating for the focal-plane tilt, the aberration dependence of the field of view is reconstructed by collecting the same image at different focal positions.

In theory, the single holographic lens of the AIT microscope is aberration corrected for just one point in the field. Away from that point, the dominant aberration is astigmatism, which is predicted to reach a magnitude of 0.35 waves PV in the corners of the visible field. The second important field-dependent aberration is coma. In the ideal alignment state, the coma magnitude is six times less than the astigmatism magnitude; reaching a peak value of 0.062 waves PV. Figure 2 shows two aberration maps calculated from the model for astigmatism and coma. Where $Z_{\mathrm{j}}$ is the coefficient of the $j$-th Zernike polynomial ${ }^{8}$, the aberration magnitudes are defined as: $\left(Z_{4}{ }^{2}+Z_{5}^{2}\right)^{0.5}$ for astigmatism, and $\left(Z_{7}{ }^{2}+Z_{8}{ }^{2}\right)^{0.5}$ or coma. Figure 2 shows that the aberration magnitude increases in all directions away from the center. Also calculated but not shown are the specific variations in the $Z_{4}$ and $Z_{5}$ coefficients that are important during alignment, for determining our position within the field. 

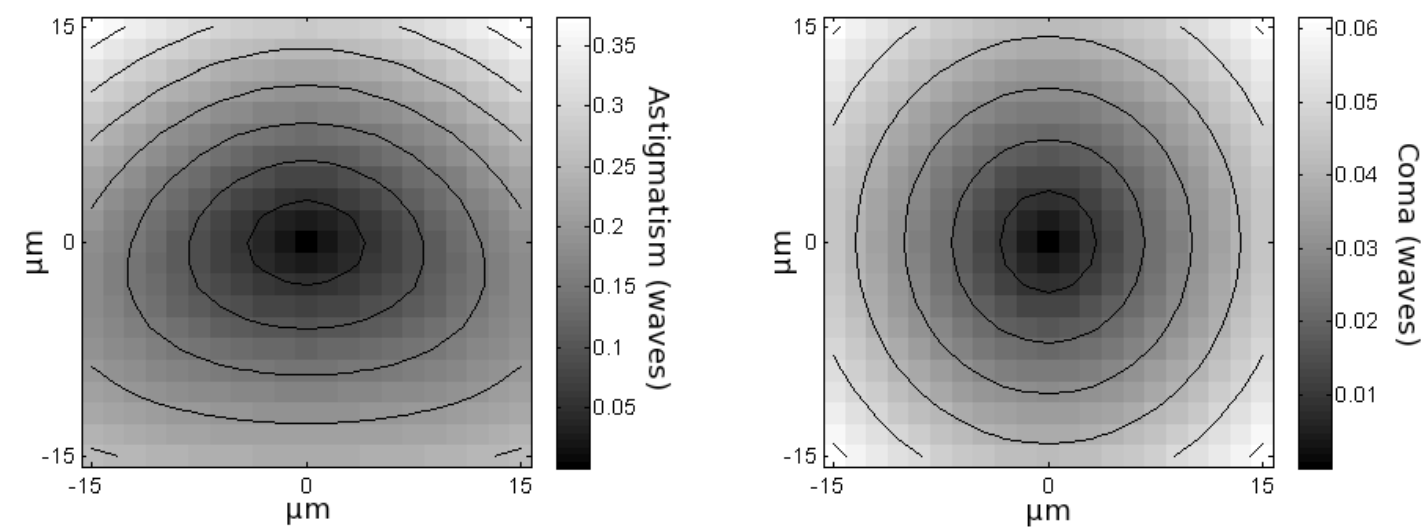

Fig. 2. Astigmatism and coma magnitude maps over the AIT's observable field of view in the case of perfect alignment. These maps refer to the configuration with $0.0875 \mathrm{NA}$ and focal length $0.750 \mathrm{~mm}$. Astigmatism is the dominant aberration of the optical system and it has a peculiar arc shape that is easily recognizable

We will show how a field of lose-pitch contacts can be used as a local aberration monitors across the field. The local astigmatism can be calculated at numerous points in the field and fit to the predicted behavior to determine the direction and amount of system misalignment.

In an optical system affected by astigmatism, the shape of the wavefront has a component described by a generic combination of the two Zernike polynomials $Z_{4}$ and $Z_{5}$

Where $\mathrm{Z}_{4}$ and $\mathrm{Z}_{5}$ are given by:

$$
A W=A \cdot Z_{4}+B \cdot Z_{5} \text {. }
$$

$$
Z_{4}=A_{022} \rho^{2}\left(2 \cos ^{2}(\theta)-1\right) \quad Z_{5}=A_{022} \rho^{2}\left(2 \sin ^{2}(\theta)-1\right)
$$

In the case of a perfect (aberration-free) system with circular aperture, the point spread function (PSF) is an Airy pattern:

$$
P S F=\frac{2 J_{1}(v)}{v} . \quad v=2 \pi r .
$$

Where $J_{1}$ is a Bessel function of the first kind and $r$ is the pupil's radius. Through focus, the shape of the PSF changes substantially but retains its circular symmetry, as shown in Fig. 3.

When astigmatism is present, the image of a point source changes its shape through focus, becoming elliptical in two orthogonal directions. The beam waist in two directions defines two displaced 'foci'. The azimuthal orientation of the astigmatism determines the direction of the major and minor axes of the ellipse, which flip, through focus.

The distance between the foci is called the astigmatic displacement (AD). Using the $\mathrm{AD}$ magnitude, and the azimuthal angle defined by the two planes the focused rays come from, it is possible to calculate the two components $A$ and $B$ of the aberrated wavefront $(A W)$. Let's consider a spherical reference wave converging towards the image. Let $S$ be the astigmatism magnitude in waves, $R$ the radius of the reference spherical wave, $a$ the radius of the exit pupil. The astigmatic displacement $A D$ is given by ${ }^{8}$ :

$$
A D=2 S \frac{R^{2}}{a^{2}} .
$$

In the AIT the entrance and exit pupils coincide, and the measurement of the focal displacement is performed by motion on the object (mask) side, rather than with the CCD detector. Let $M$ be the magnification of the system, $\lambda$ the wavelength, and $\alpha$ the angle defined by the arctangent of the ratio between $B$ and $A$ : the astigmatism in waves can be calculated with the expression

$$
S=A D^{\prime} \frac{M^{2} a^{2}}{2 R^{2} \lambda} . \quad A=S \cos (\alpha), \quad B=S \sin (\alpha) .
$$


Where $A D^{\prime}$ is the astigmatic displacement measured in the object space. To calculate the astigmatic wavefront error, we need to know the astigmatic displacement $A D^{\prime}$ and the angle $\alpha$. Both quantities can be obtained analyzing the through focus images of a sub-resolution square contact.

\section{A}
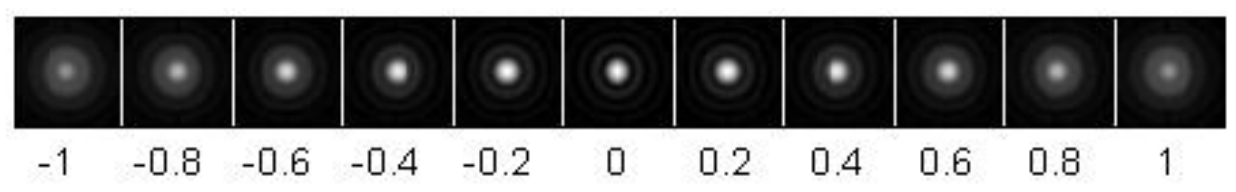

B

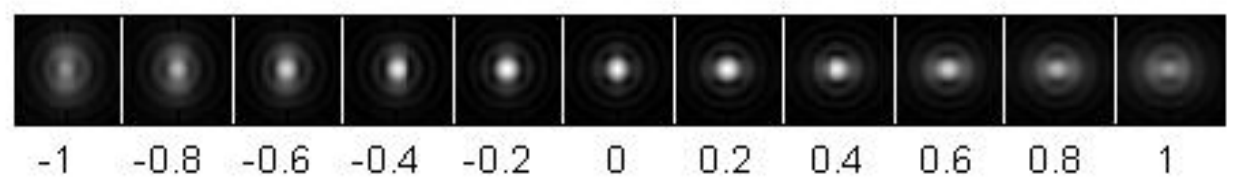

C

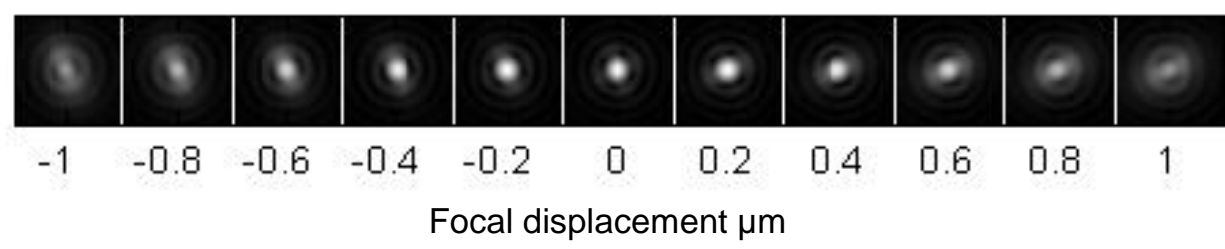

Fig. 3. (A) Point spread function of an unaberrated system through focus. (B) Point spread function affected by 0.3 waves of $\mathrm{Z}_{4}$ astigmatism. (C) Point spread function affected by 0.3 waves of $\mathrm{Z}_{4}+\mathrm{Z}_{5}$ astigmatism.

We will describe here the algorithm we developed to measure the astigmatic displacement and the astigmatism components ratio. The resolution limit of an optical instrument with coherent illumination is given by ${ }^{8}$ :

$$
Y=0.82 \frac{\lambda}{N A} \text {. }
$$

The 'contact field' used in the aberration analysis is a grid of small, bright squares, loosely spaced in a dark field. The squares measure 175-nm-wide on the mask, and are spaced with a 1:4 square to space ratio. Using the AIT's 0.0875 NA zoneplate lens, the resolution, $Y$, is approximately $126 \mathrm{~nm}$, according to the previous definition. Using contacts that are only fractionally larger than the resolution means that the image is affected by the finite solid angle of the entrance pupil and loses most of its square appearance, as shown in Fig. 4. For the purpose of this calculation the image of a single contact can be approximated with a two-dimensional Gaussian surface:

$$
I(x, y)=I_{0} \exp \left(-\left(\frac{\tilde{x}-\tilde{x}_{0}}{\sigma_{x}}\right)^{2}-\left(\frac{\tilde{y}-\tilde{y}_{0}}{\sigma_{y}}\right)^{2}\right) .
$$

The selection of this function captures the elliptical behavior induced by astigmatism, when it is present, while filtering other asymmetric intensity components. The validity of this assumption has been tested through simulation described later.

The coordinates are expressed as a simple rotation of the image coordinate system:

$$
\left(\begin{array}{l}
\tilde{x} \\
\tilde{y}
\end{array}\right)=\left(\begin{array}{cc}
\cos (\alpha) & -\sin (\alpha) \\
\sin (\alpha) & \cos (\alpha)
\end{array}\right)\left(\begin{array}{l}
x \\
y
\end{array}\right) .
$$



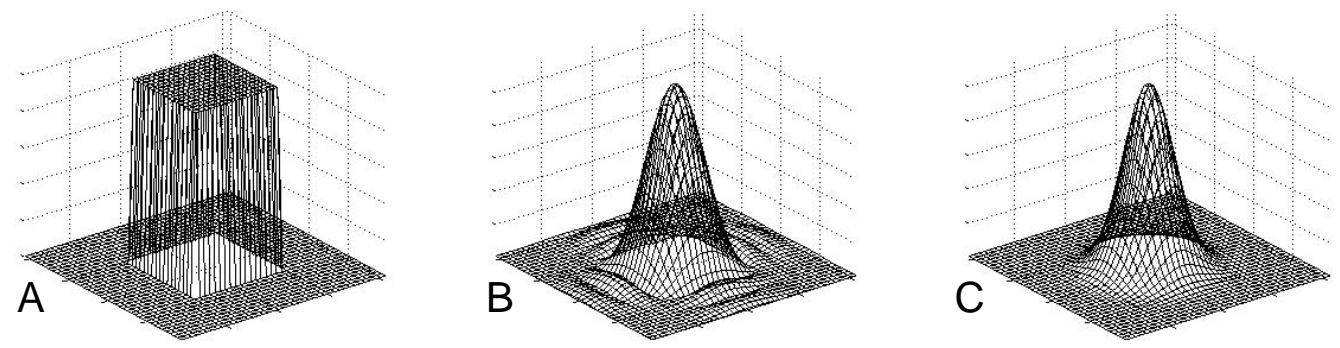

Fig. 4. (A) Intensity mesh of a $175 \mathrm{~nm}$ side square contact. (B) Image of a $175 \mathrm{~nm}$ side square contact through an optical system with NA $=0.0875$ and $\lambda=13.4 \mathrm{~nm}$. (C) Two-dimensional Gaussian fitting of the image intensity shown in (B).

Fitting the model to the collected images, we obtain an estimate for the center of the contact $\left(x_{0}, y_{0}\right)$, the angle $\alpha$, and the half widths along the tangential and sagittal planes $\sigma_{\mathrm{x}}$ and $\sigma_{\mathrm{y}}$.
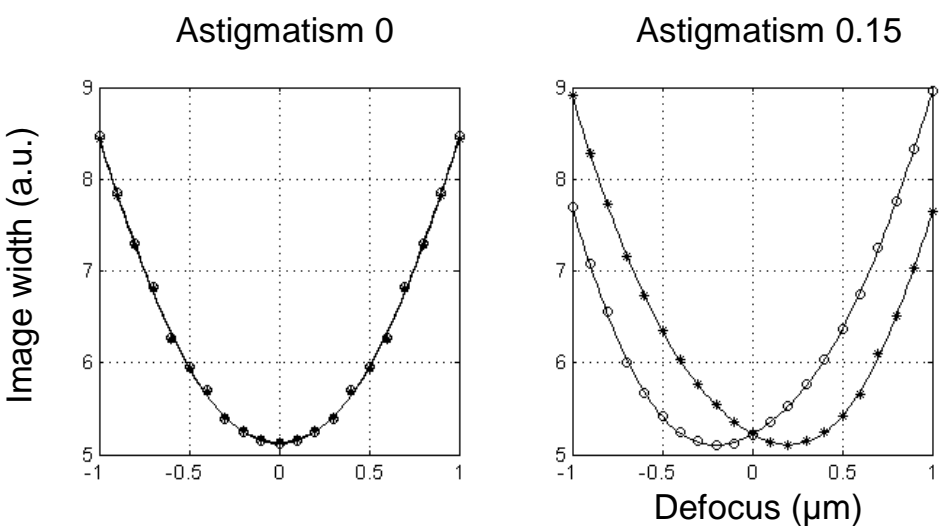

Astigmatism 0.3 waves

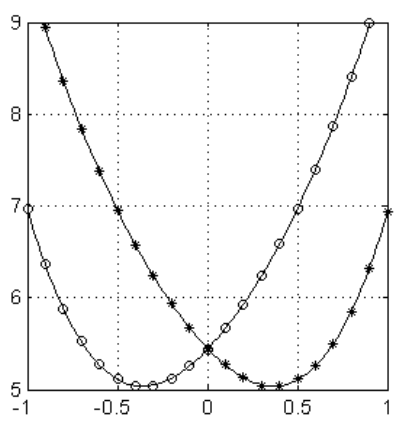

Fig. 5. The determination of the astigmatic displacement (AD) in three different cases. Out of focus, the image width increases. The plots show the evolution of $\sigma_{\mathrm{x}}$ (circles) and $\sigma_{\mathrm{y}}$ (stars) as the focal position is changed; the solid lines represent a $4^{\text {th }}$ order polynomial fit to the measured values. The values on the vertical axes are expressed in camera pixels ( $15.3 \mathrm{~nm} / \mathrm{px}$ in object space). The AD is measured as the separation distance of the two minima. Note also that the size of the image at best focus (Defocus=0 position) increases with the astigmatism magnitude.

To evaluate different fitting methods we generated simulated contact images through focus, with noise and added astigmatism of various magnitudes and directions. We tested two fitting methods to compare their computation time and stability. The first technique is a direct-search method based on the Nelder-Mead Simplex algorithm ${ }^{9,10}$. The advantage of this method is that it takes into account the whole surface at one time without the need of any data pre-processing and is therefore very robust; its downside is that it is potentially slow and computationally cumbersome. The second method relies on a simplification of the model. We evaluated the center of the image with a recursive algorithm for the image centroid determination. Then we calculated the direction of maximum elongation using a simple search. The major and minor axes of the cross-section ellipse are found by fitting two orthogonal Gaussian functions curves to the image's intensity profile, measured along the directions of maximum and minimum elongation. In practice, we found this method to be as stable as the first, and much faster. This time difference is attributable to the fact that the second method uses the standard Levenberg and Maquardt least squares method ${ }^{9}$ to perform the fit.

In presence of astigmatism, the aerial image's minimum widths along the sagittal and tangential plane correspond to the position of the tangential and sagittal foci respectively. We measured the evolution of $\sigma_{\mathrm{x}}$ and $\sigma_{\mathrm{y}}$ and estimated the focal displacement of the two minima performing an approximation of the data with a $4^{\text {th }}$ order polynomial as shown in Fig. 5 . From the AD, the NA and the wavelength, we were able to calculate the astigmatism magnitude. The angle $\alpha$ was obtained directly from the fit and allowed us to evaluate the magnitude of the single components of the astigmatism. 


\subsection{Simulation and error evaluation}

To verify the assumptions we made in the development of these fitting methods, we studied the measurement errors resulting from a variety of input conditions, using simulated image data.

In the development of this algorithm we made two main assumptions. The first one is the absence of significant contributions to the wavefront by other off-axis aberrations that could bias the shape of the contacts in a specific way. The second assumption is that the contact we used is close enough to the resolution limit that the Gaussian model we chose to fit the intensity profile is still a good approximation. The first assumption was made possible by a raytracing simulation, which showed that when the system is close to the correct alignment, the coma magnitude is much smaller than the astigmatism magnitude. To verify the second assumption we simulated the image of a single square contact with the same size as the contacts we used in the actual experiment.

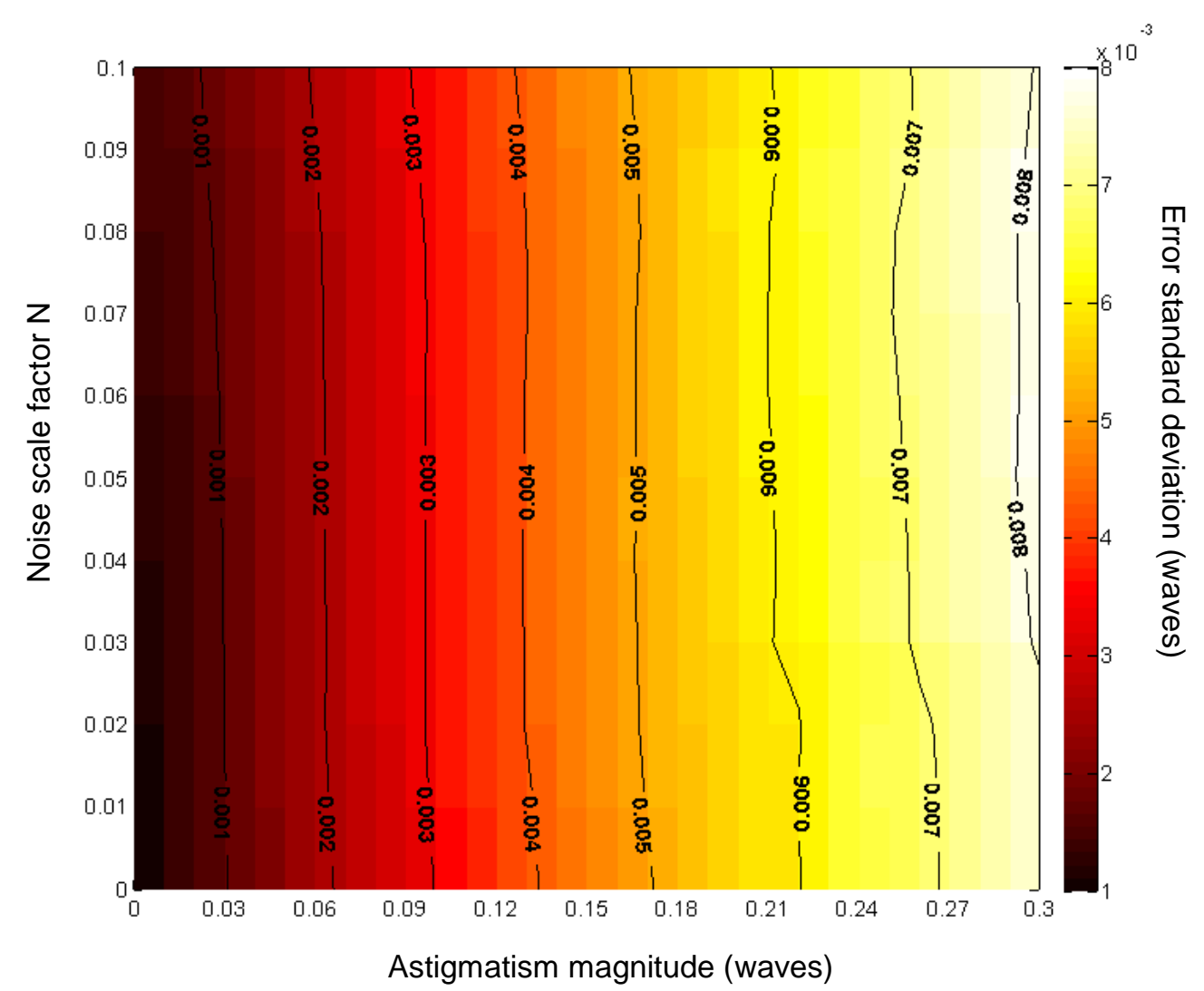

Fig. 6. This error map shows the standard deviation error for the astigmatism measurement algorithm as a function of the noise level and the astigmatism magnitude. In this map the directions of the astigmatism, or the ratio between the coefficients of $Z_{4}$ and $Z_{5}$, has been averaged over all azimuthal angles.

Our parameter space included both the magnitude and the direction of the astigmatism. In each case, we used our algorithm to measure the $\mathrm{AD}$ for comparison with the input value. We learned that the square shape of the contact does affect the astigmatism evaluation in subtle ways. However, this effect is small enough to be negligible in the range of small astigmatism values we are used to working with in practice. In addition, we performed the calculations in the presence of increasing amounts of photon shot noise, following the Poisson distribution, for varying input intensity levels. For convenience the images are scaled before being fed to the astigmatism calculation algorithm. The scaling factor applied is such that, in each through focus series, the image in best focus has a peak intensity of 1 . We added photon shot noise with a scale factor $N$ in order to have peak signal to noise ratio of $1 / N^{2}$. 
The results of the simulation are summarized in Fig. 6 where we show the standard deviation of the calculated astigmatism as a function of the noise scale factor $N$ and the magnitude of the astigmatism introduced in the wavefront. The simulation shows that the error depends on both parameters and increases for high astigmatism values, however the relative error magnitude is less than $10 \%$ and decreases as the astigmatism value increases. The robustness of the method, shown by the low absolute error value, is a result of the fact that each astigmatism value is obtained averaging a considerable amount of information. The Gaussian fitting is performed on 100 by 100 pixels sub-images. A series of 21 images through focus is used and the astigmatic displacement is finally obtained with a $4^{\text {th }}$-order polynomial fitting. Nevertheless we must remember that this calculation doesn't take into account the effect of the presence of other aberrations that could compromise the stability of this algorithm, for this reason these values have to be considered as lower limit of the error. An evaluation of the effect of coma and spherical aberration is required to obtain a further characterization of this method.

\section{EXPERIMENTAL DATA AND ALIGNMENT}

The alignment procedure consists of progressive adjustments of the zoneplate's position and rotation angle. The feedback to tune these degrees of freedom is provided by the measurement of the astigmatism value over the field of view of the AIT. In essence, we are trying to align the center of the CCD camera's observable area with the zoneplate's sweet spot, where the aberrations are minimum. To measure the astigmatism over the whole field of view we used a reticle with several arrays of square contacts, each one with a different size. We chose an array containing $175 \mathrm{~nm}$ squares spaced (5-1). This choice is compatible with the simulation previously described, in addition it allowed us to maintain a good signal to noise ratio and to fill the whole field of view in a single shot (figure 7).

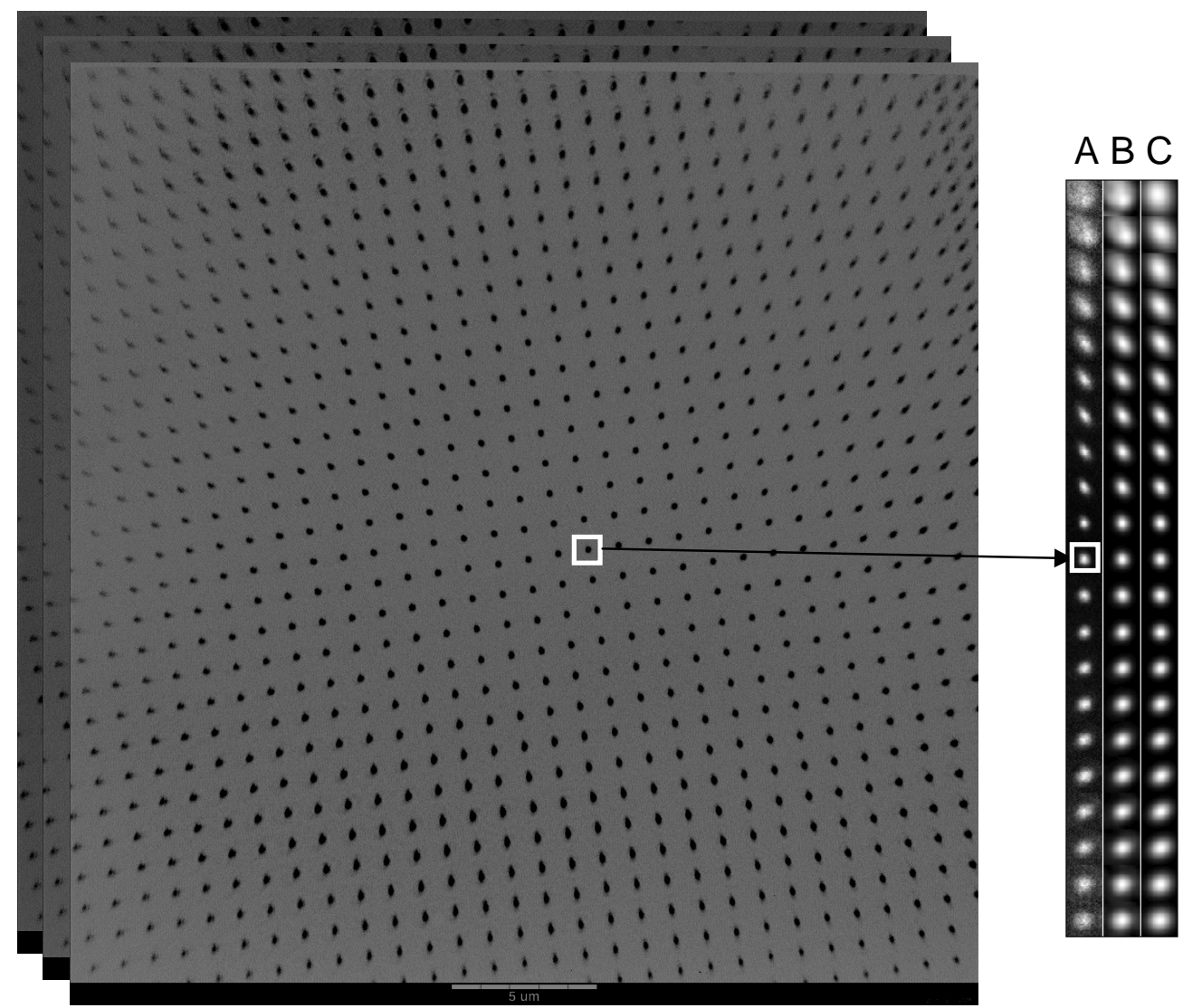

Figure 7 AIT image of an array of $175 \mathrm{~nm}$ size square contacts spaced 5-1. The colors on the image on the left panel are inverted to look better in the printed version (black corresponds to high intensity). On the right panel we show a single contact through focus. Column A shows the raw data, column B shows the same data processed with a lowpass filter and column $\mathrm{C}$ shows the two dimensional Gaussian fitting used in the algorithm for the astigmatic displacement calculation. 
In figure 8 we show an example of alignment performed in four steps tweaking the zoneplate rotation angle $\theta$. Starting from a poor alignment condition we followed astigmatism map evolution until it matched the shape of the theoretical map shown in figure 2 .

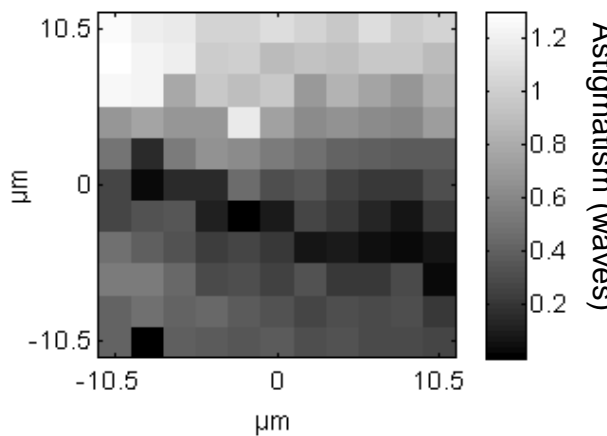

A

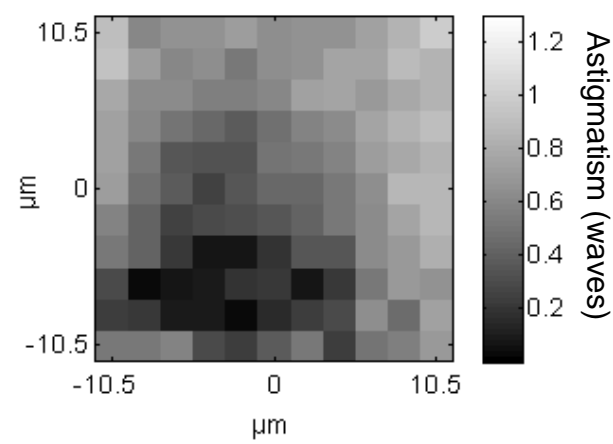

C

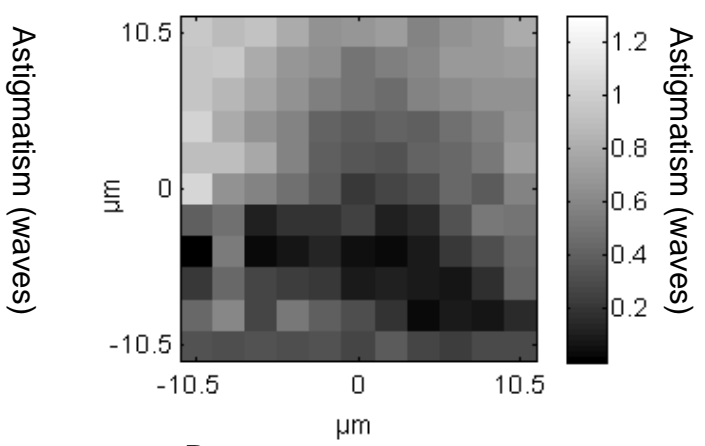

B

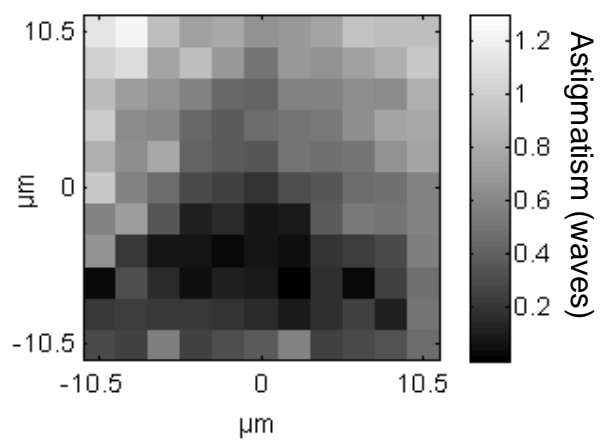

D

Fig. 8. Measured astigmatism maps in four successive alignment steps. (A) We started with the system in a poor alignment state, the low astigmatism values are arranged along a line and the lowest astigmatism is on the bottom right just outside the field of view. (B) We rotated the zoneplate lens and the sweet spot moved back into the observable field. (C) Further rotation of the lens moved the minimum astigmatism into the bottom left of the observable field. (D) The last step. We reversed the lens rotation a small amount to bring the center the arc-shaped astigmatism minimum to the right.

Figure 9 shows the how the new alignment feedback methods have led to direct improvements in image quality. Shown are three series of contact images through-focus, recorded several months apart, spanning almost one year. 


\section{CONCLUSIONS}

We have developed and tested an effective method for optimizing the alignment of the AIT. Using a large array of darkfield contacts as the mask pattern, and observing their behavior through focus, we developed a straightforward method to accurately extract the local astigmatism magnitude and direction. The alignment method is based on the comparison of the measured aberrations across the observable field of view to a prediction of the zoneplate's ideal behavior, based on a raytracing model. The method for astigmatism calculation relies on fitting the elliptical shape of the contact images, and is highly robust in the presence of noise. This algorithm enables us to visualize the position and the size of the alignment sweet spot, the area within the field of view where the aberrations are minimized and the data will be most reliable.

The result of this improved imaging has been higher resolution, increased image contrast, and more accurate aerial image measurements. One drawback of the current technique is the reliance on contact fields, which are not available on every mask. Future alignment feedback methods may use arbitrary mask patterns and include aberrations beyond astigmatism.

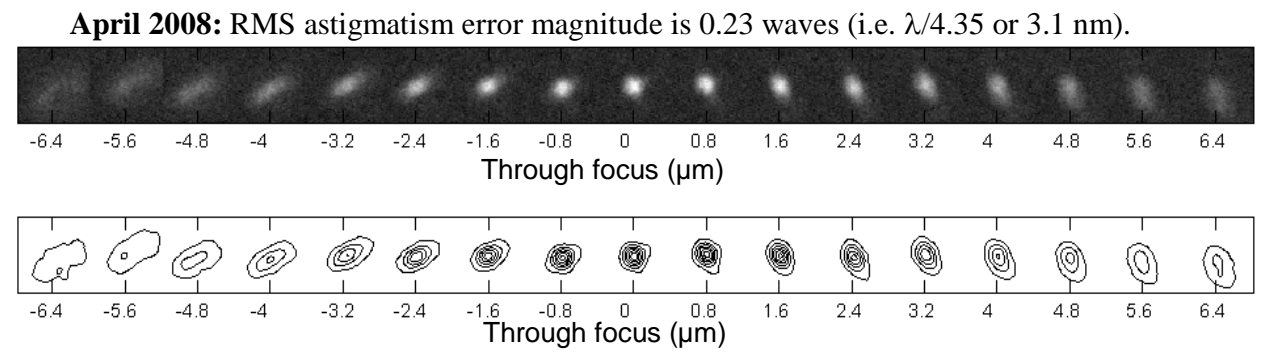

August 2008: RMS astigmatism error magnitude is 0.08 waves (i.e. $\lambda / 12.5$ or $1.1 \mathrm{~nm}$ ).

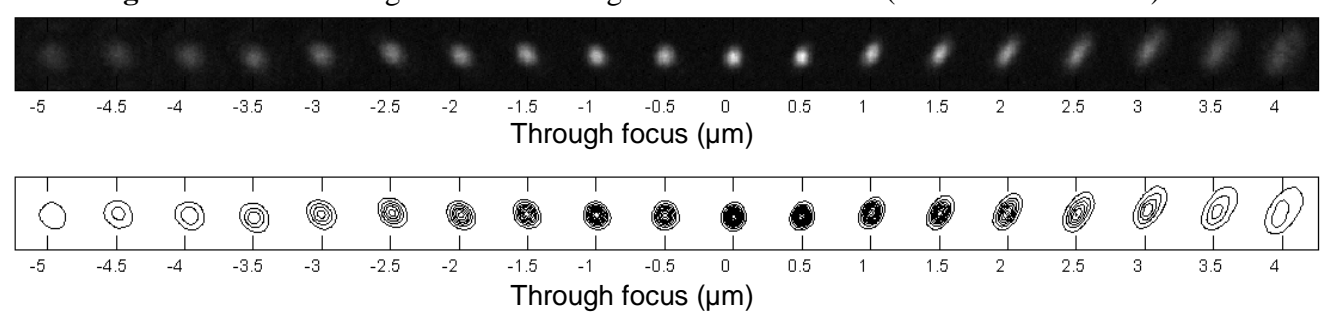

January 2009: RMS astigmatism error magnitude is within the measurements uncertainty.
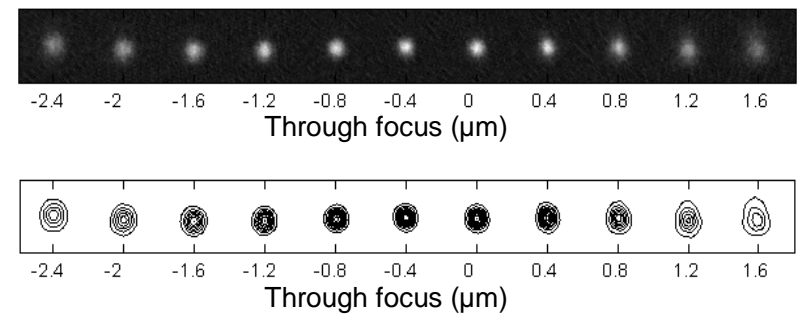

Fig. 9. Progress in the AIT imaging alignment from April 2008 to January 2009 is summarized here. Three sets of contact images through focus show the behavior of a single 175-nm square contact (mask dimensions) in the middle of the AIT's field of view. The progressive improvement in the aberration magnitude has been quantified, measuring the astigmatism across the field of view. Following the most recent alignment, the calculated astigmatism in the sweet spot is zero, within the uncertainty of the measurement the measure $(<0.001$ waves). 
This work was performed under the auspices of the U.S. Department of Energy by University of California Lawrence Berkeley National Laboratory, and was funded by SEMATECH. Research was conducted by scientists from the Materials Sciences Division of Lawrence Berkeley National Laboratory at the Advanced Light Source with support from the ALS Division. This work was funded by Sematech, Inc., Contract No. 401832-NY through the U.S. Department of Energy under Contract No. DE-AC02-05CH11231.

\section{REFERENCES}

[1] K. A. Goldberg, I. Mochi, P. P. Naulleau, H. Han, and S. Huh, "Benchmarking EUV mask inspection beyond 0.25 NA." SPIE, 7122, 71222E (2008).

[2] C. H. Clifford, S. Wiraatmadja, T. T. Chan, A. R. Neureuther, K. A. Goldberg, I. Mochi, T. Liang. Comparison of fast 3D simulation and actinic inspection for EUV masks with buried defects. SPIE, These proceedings, 7271-51.

[3] P. Dirksen, C. A. Juffermans, A. Casper; R. J. Pellens; M. Maenhoudt; P. Debisschop, "Novel aberration monitor for optical lithography" SPIE 3679, p. 77-86, Optical Microlithography XII. (1999).

[4] F. X. Zach, C. Lin, and J. P. Kirk, "Aberration analysis using reconstructed aerial images of isolated contacts on attenuated phase-shift masks." SPIE 4346, 1362 (2001).

[5] N. R. Farrar, A. H. Smith, D. R. Busath, and D. Taitano, "In-situ measurement of lens aberrations" SPIE 4000, 18 (2000).

[6] P. Dirksen, J. Braat, A. J. E. M. Janssen, A. Leeuwestein, H. Kwinten, and D. Van Steenwinckel, "Determination of resist parameters using the extended Nijboer-Zernike theory" SPIE 5377, 150 (2004).

[7] K. A. Goldberg, P. P. Naulleau, A. Barty, S. B. Rekawa, C. D. Kemp, R. F. Gunion, F. Salmassi, E. M. Gullikson, E. H. Anderson, H.-S. Han, "Performance of Actinic EUVL Mask Imaging Using a Zoneplate Microscope", SPIE, 6730, p. 67305E, (2007).

[8] M. Born, and E. Wolf, [Principles of Optics], 6th Ed., Pergamon Press, New York, (1980).

[9] W. H. Press, S. A. Teukolsky, W. T. Vetterling, B. P. Flannery, [Numerical recipes in C], Cambridge University Press, Cambridge, New York, Port Chester, Melbourne, Sydney, 408-412 (1992).

[10] J. A. Nelder, and R. Mead, Computer Journal 7, 308-313 (1965). 